\title{
تطبيق إدارة الجودة الشاملة في التعليم الجامعي: دراسة حالة في كلية الدراسات الإسلامية جامعة المحمدية مالانية المانج.
}

\author{
لبنى أحمد شهوب وعبد المالك كريم أمر الله \\ جامعة مولانا مالك إبراهيم الإسلامية الحكومية أمرالية
}

Email:lash772002@yahoo.com, amkamutia@gmail.com

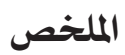

يعد علم الجودة في العصر الحالي من أهم العلوم الإدارية الحديثة التي تساهم بشكل

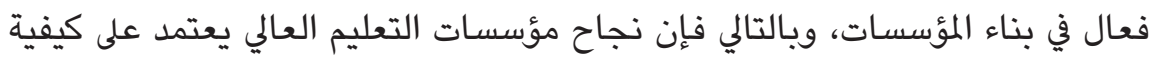

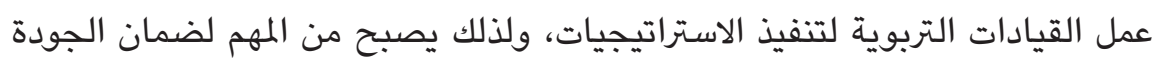
التحسين المستمر للتعليم العالي.

هدفت هذه الدراسة إلى تحديد تطبيق إدارة الجودة الشاملة في كلية الدراسات الإسلامية في

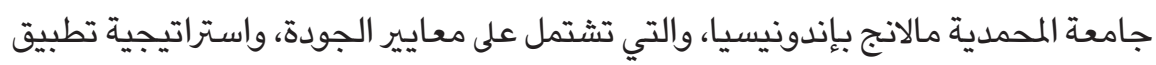

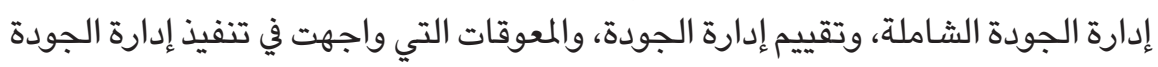
وكيفية تطوير الجودة الشاملة في كلية الدراسات الإسلامية في جامعة المحمدية الماتية مالانج.

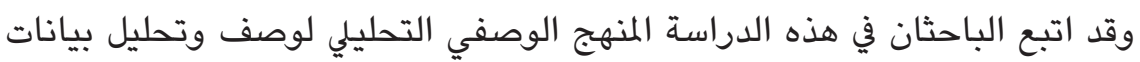

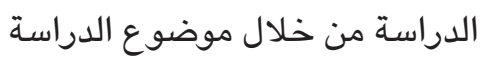

ومن أهم النتائج التي توصلت إليها الدراسة أن معايير جودة التعليم في جامعة المحمدية

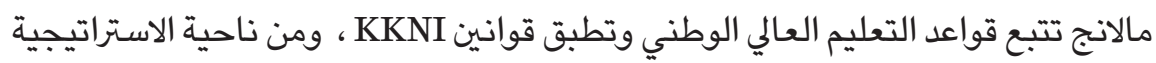

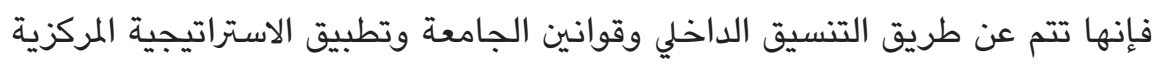

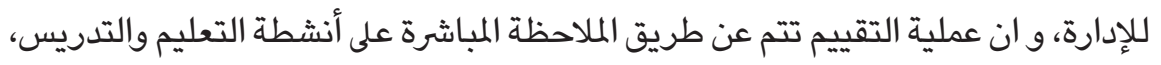

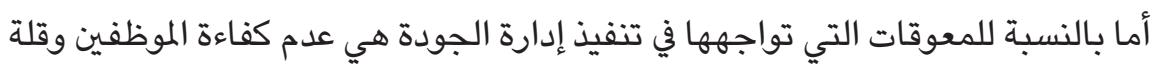




$$
\begin{aligned}
& \text { الوعي لتطبيق المعايير الجديدة للجودة، ويتم تطوير إدارة الجودة من خلال تطبيق قواعد } \\
& \text { التعليم بشكل واضح من قبل الجهات المعنية ومراقبة تطبيقها. }
\end{aligned}
$$

\section{Abstract}

The success of an institution or university depends on how the leader educates and implements the strategy. Therefore, an education strategy must be developed by university in every nation worldwide. This research aims to know the implementation of TQM in the Faculty of Islamic Study at Muhammadiyah University, Malang, Indonesia, which include Quality standard, TQM implementation strategy, TQM evaluation, and the obstacles of TQM implementation. This research employs descriptive analysis. The instrument of data collection is by interview, documentation and observation. The most important findings from this research is the quality standard in Muhammadiyah University Malang follows the regulation of National Higher Education and formulated based on KKNI. The strategy of TQM implementation was conducted from the central of quality management, while the process of quality evaluation was conducted directly from the teaching and learning activities. The obstacles faced by the TQM implementation is the lack of professionals and the lack of awareness to implement the new quality standard. In addition, the development of TQM is done by implementing the education policy firmly and it must be controlled continuously.

Keywords: quality management, higher education, quality standard, education policy

\section{المقدمت}

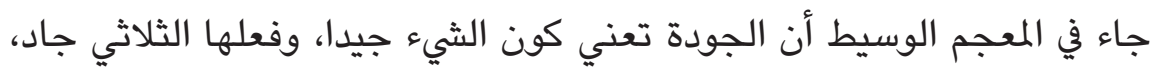

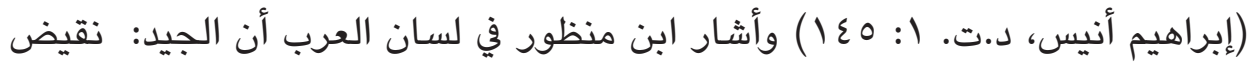

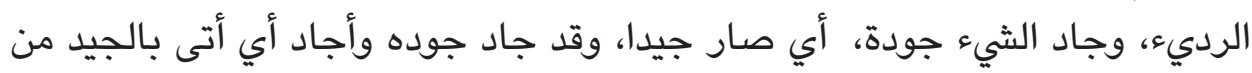

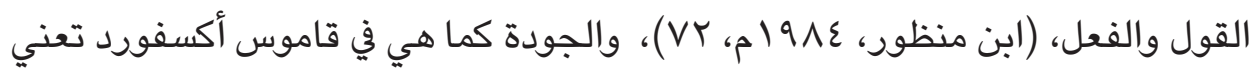

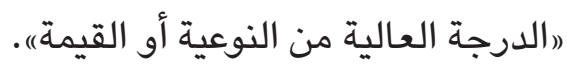

والجودة كمصطلح Quality كلمة مشتقة من الكلمة اللاتينية (كواليتاس) التي

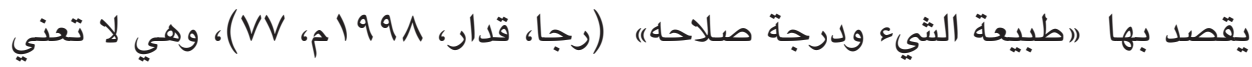

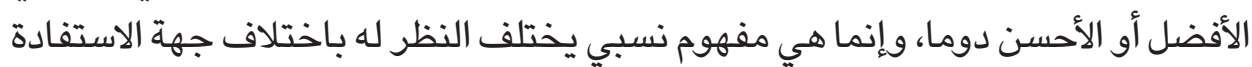

$$
\text { منه سواء كان (الزبون، المصمم، المجتمع، المؤسسة .. الخ). }
$$




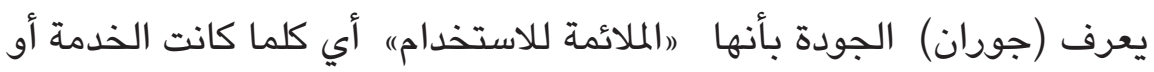

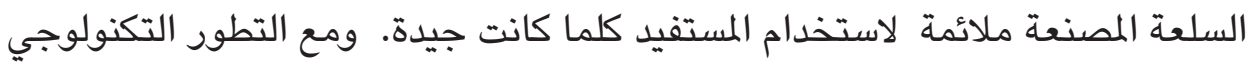

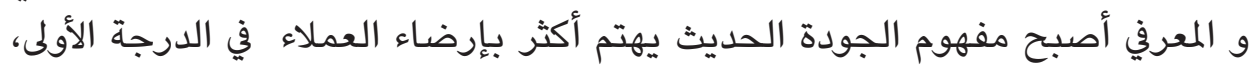

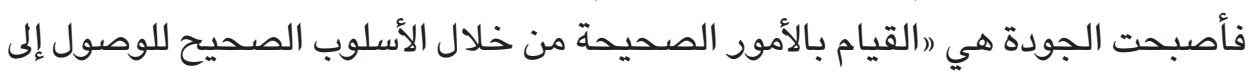

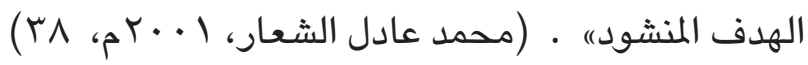

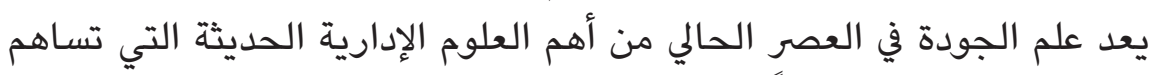

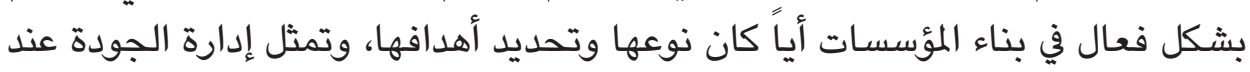

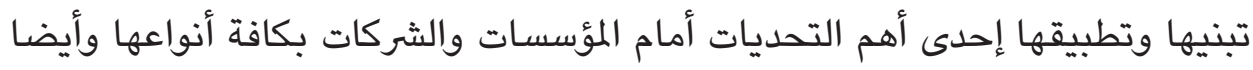

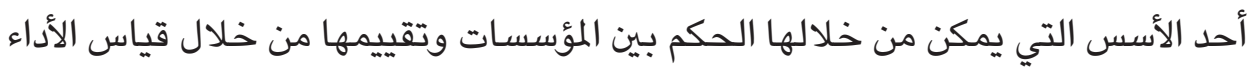

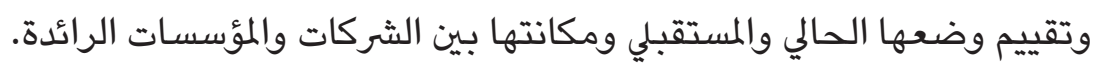

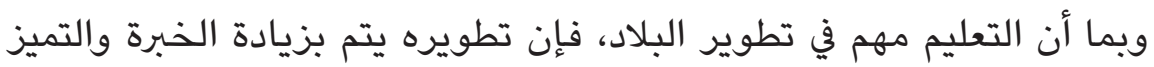

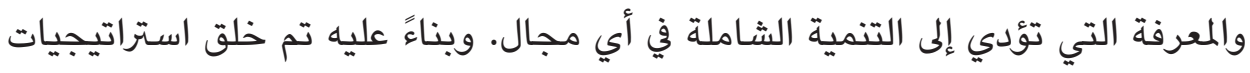

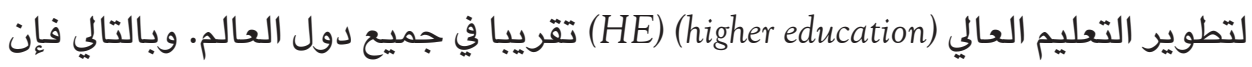

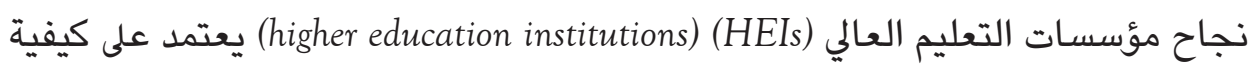
عمل القيادات التربوية لتنفيذ الاستراتيجيات، ولذلك يصبح من المهم لضمان الجودان لتودة التحسين المستمر للتعليم العالي.

وبالاطلاع على الدراسات في هذا المجال نجد أن معظم تطبيق إدارة الجودة له الهاله

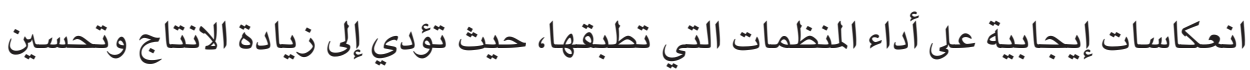

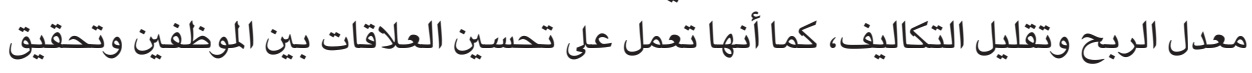

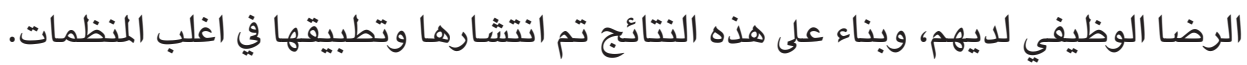

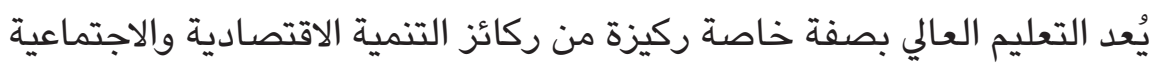

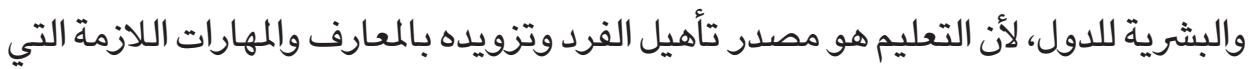

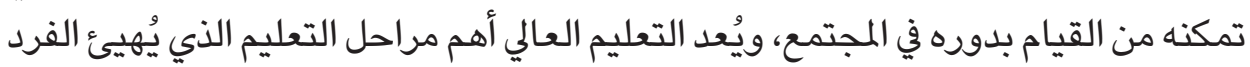

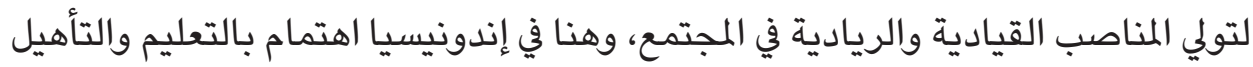

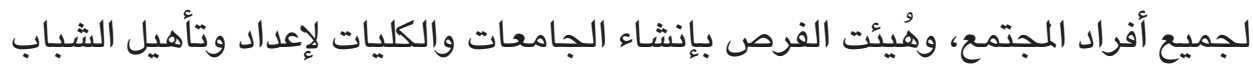

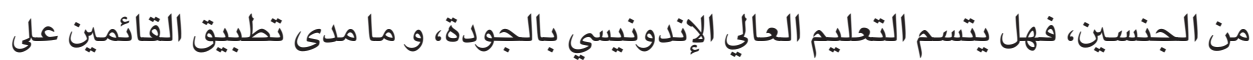

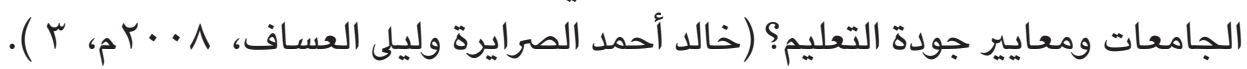


ويالتالي فقد قامت الباحثة بهذه الدراسة لمعرفة مدى اهتمام وتطبيق المؤسسات

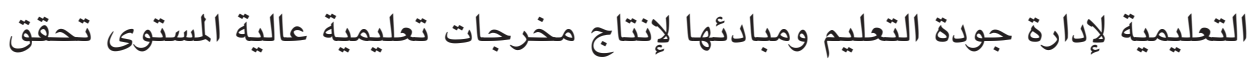

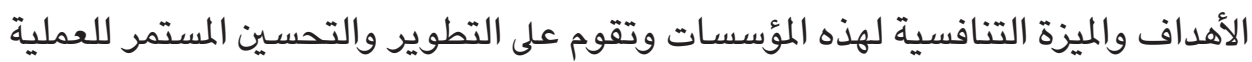

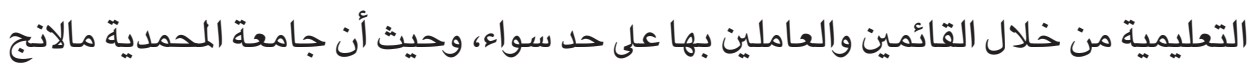

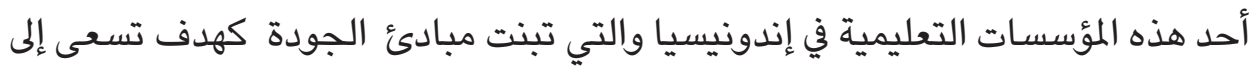

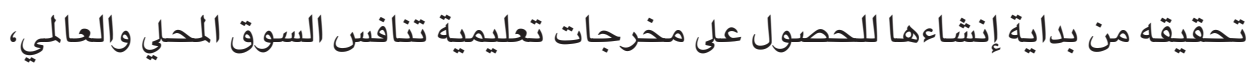

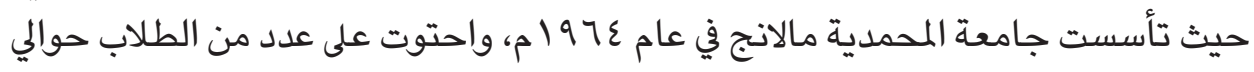

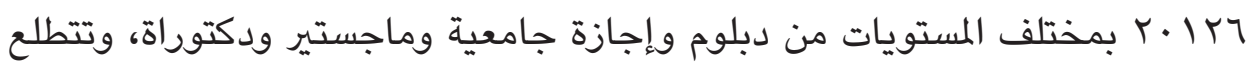

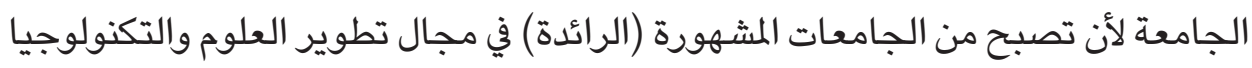
والفن على أساس القيم الإسلامية. وتعدّ إدارة جودة التعليم كمفهوم إداري حديث استحوذ على الاهتمام الكبير من

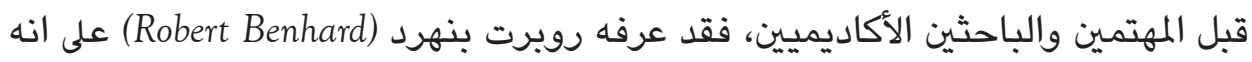

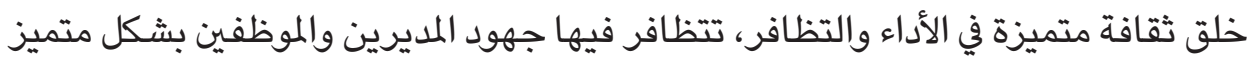

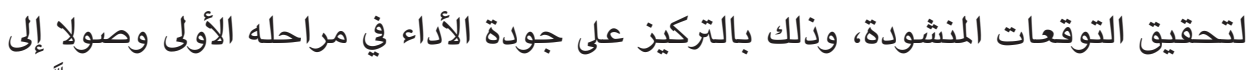

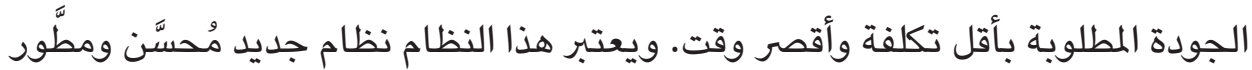

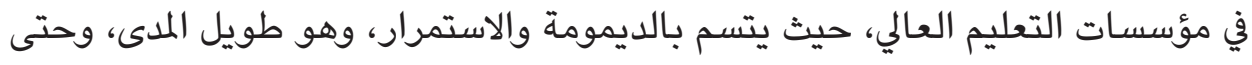

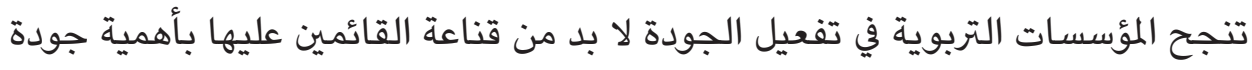

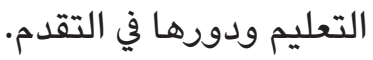
كما يتطلب تطبيق إدارة جودة التعليم توفير القناعة لدى رئاسة المؤسسات بأهمية

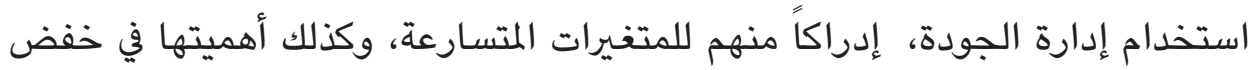

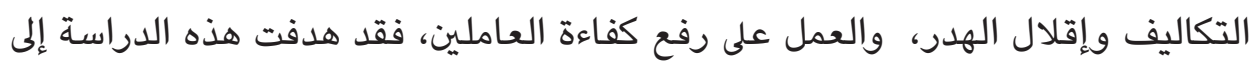

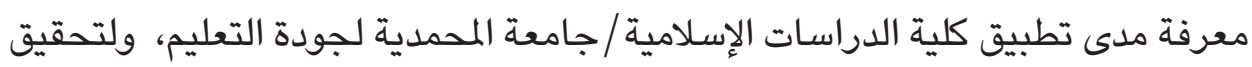
هذا الهدف ستقوم الباحثة بتصميم استبانة، وتوزيعها على مجتمع الدراسة المتمثلة في المدير ورؤساء الأقسام وأعضاء هيئة التدريس في الكلية . 
مناقشت النتائج

\section{المعايير المتبعت في جامعتم المحمديتتمالانج:}

تعتبر معايير الجودة العناصر التي يتم الحكم في ضوئها على مدى تحقيق الأهداف الخاصة بالجودة، وقد دخلت المعايير مختلف المجالات التجارية والصناعية في العقد الأخير

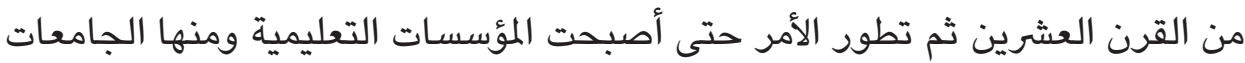

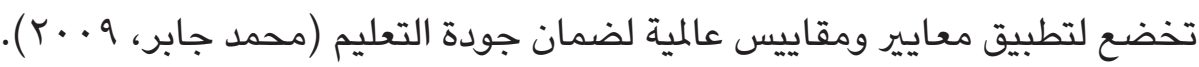
ومن ثم سارعت مختلف الجامعات بالعديد من دول العالم بتبني فكر الجودة في

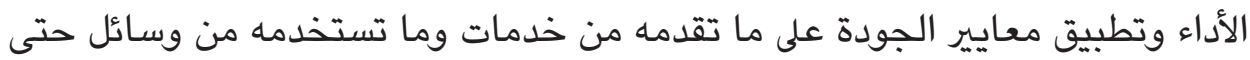
تؤدى رسالتها كمؤسسات تربوية فاعلة في المجتمع.

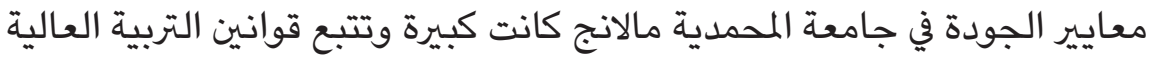

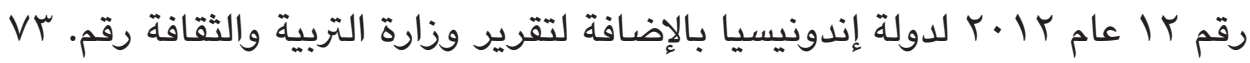

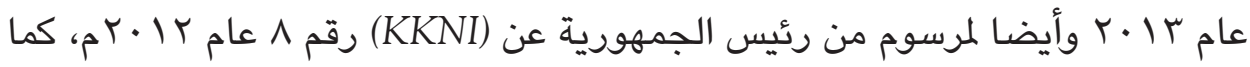

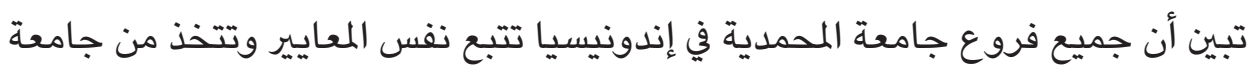

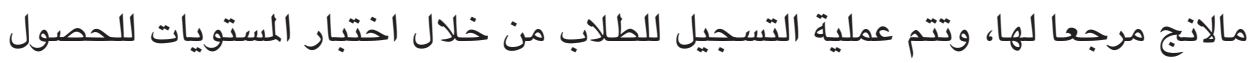

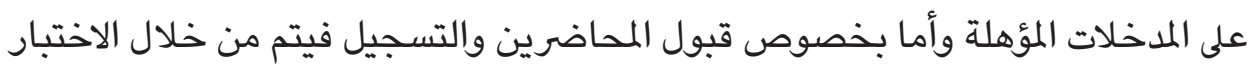

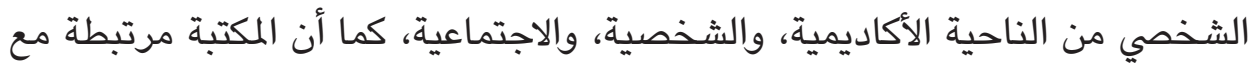
المكتبة المركزية الدولية في تحديث المعلومات. ومن خلال الملاحظة أثناء إجراء الدراسة والاطلاع على المعات المستندات والوثائق في المكاتب

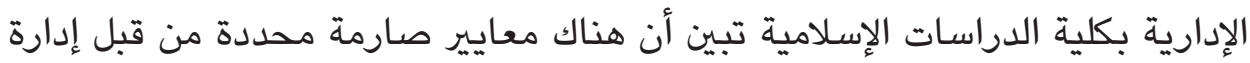

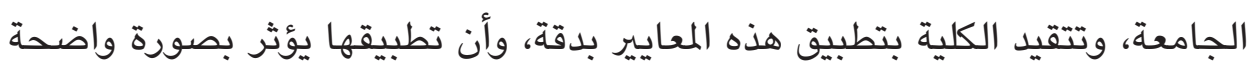
على مستوى الجامعة ومدى تطوره ورقيه.

الاستراتيجيات المتبعت في جامعت المحمديت مالانج:

يعد التخطيط في التعليم العالي أحد أهم وظائف الإدارة في المؤسسات الحديثة،

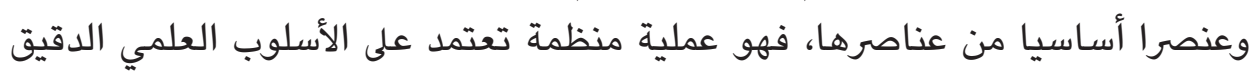

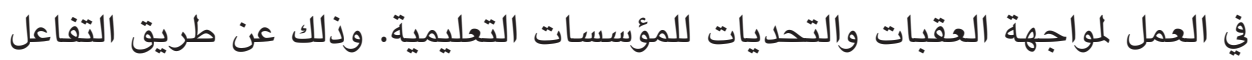

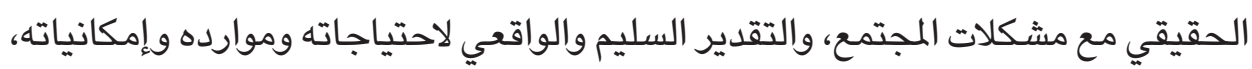

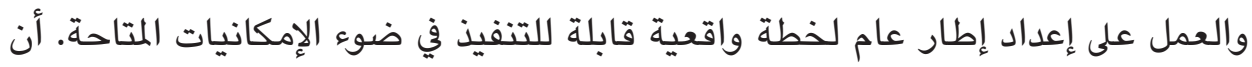




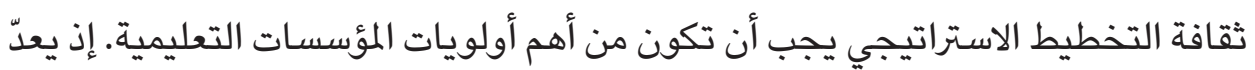
التخطيط الاستراتيجي في الجامعات ثقافة وأسلويا جديدا في التفكير والعمل وفق التقات الرؤي التئي والأهداف الاستراتيجية لكل جامعة هيئة اعتماد مؤسسات التعليم العالي 9 × • ب م.

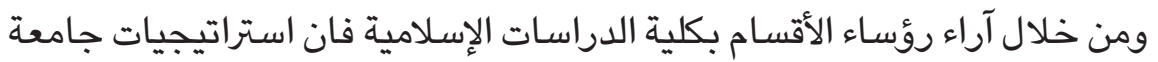

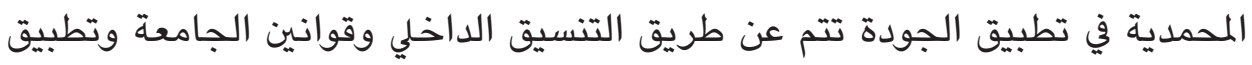

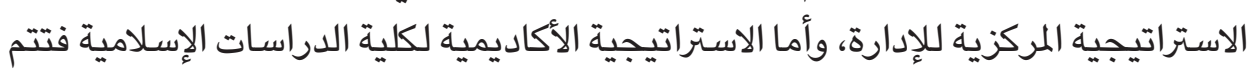
على أيدي المسؤولين في الكلية والأقسام حتى لا تحد من الابتكار الاستراتية وتنميته.

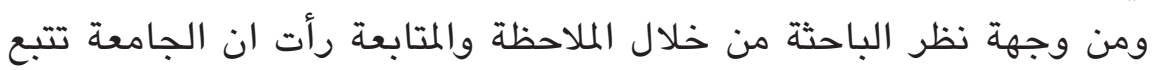
استراتيجيات ممتازة لتطبيق الجودة وهو واضح من خلال تطور مستوى الجامعة إلى مهن مستويات أفضل عاما بعد عام. معتاز لتطيق

\section{عمليت تقويم الأداء المتبعت في جامعت المحمديتشمالانج:}

يقصد بالتقويم التوصل إلى أحكام محددة للأنشطة والبرامج الجامعية من خلال استخدام بعض المقاييس المرجعية التي تساعد على فهم وإدراك العلاقة بين مختلف

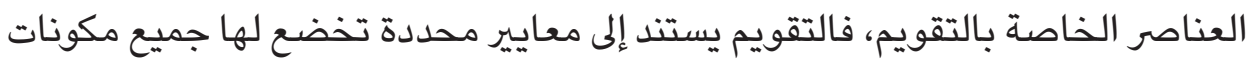
العمل الجامعي التي يمكن قياسها بحيث يمكن من خلال هذه المعايير الحكم على أداء

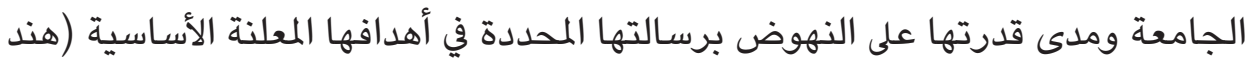

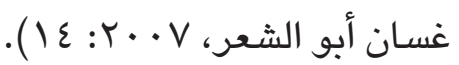
ومن خلال آراء المسؤولين بقسم الجودة وكلية الدراسات الإسلامية بجامعة المحمدية

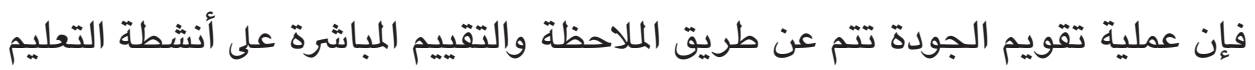

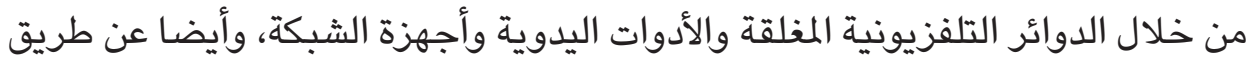

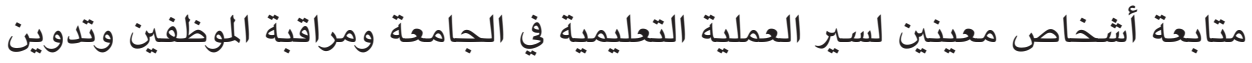
الملاحظات اليومية، وفي النهاية يتم معالجة التقصير باستدعاء المعنيين واخضاعهم لديه لدورات

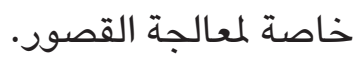
ومن خلال الملاحظة لعملية التقويم من قبل الباحثين رأيا أن العملية تتم بصورة

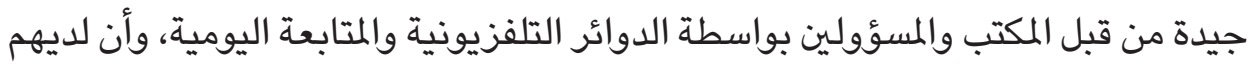

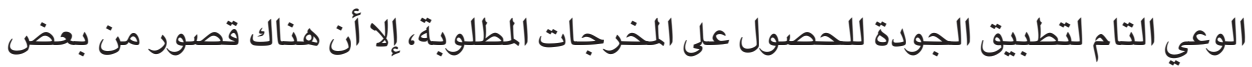

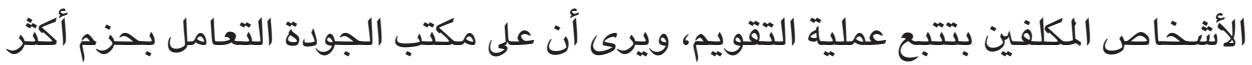
مع الأشخاص المكلفين. 


\section{معوقات تطبيق جودة التعليم في جامعت المحمديتت مالانج:}

ذكر في العديد من الأدبيات المختصة أن هناك عقبات تقف في طريق تطبيق المؤسسات

الجامعية لإدارة الجودة الشاملة كما تشير الأدبيات إلى أن هذه العقبات تتباين تبعا إلى تلى

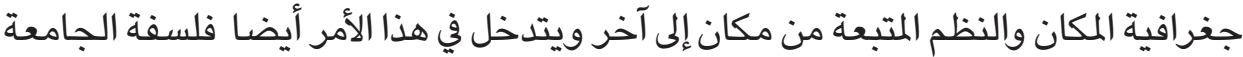

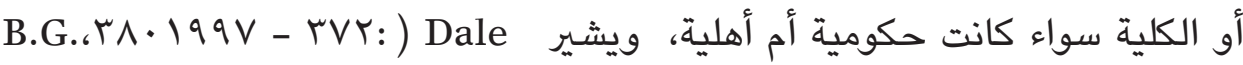
(Dale،R.J. Boaden

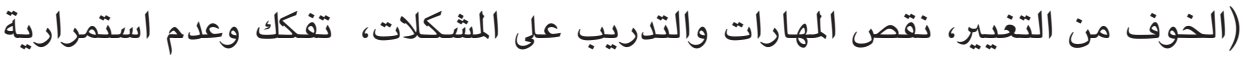

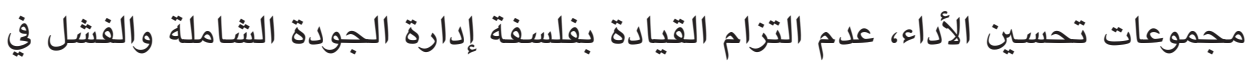

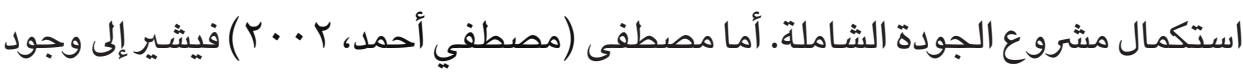

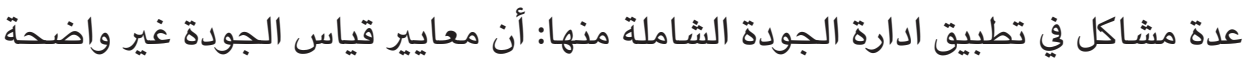

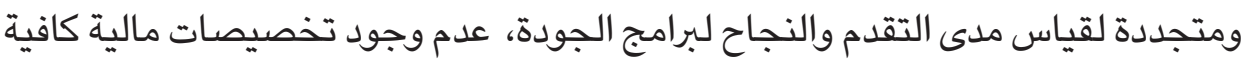
لتطبيق إدارة الجودة، قلة جهود التوثيق وتسجيل الإجراءات والنتائج •

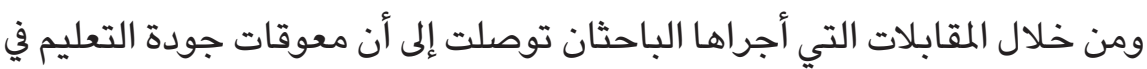

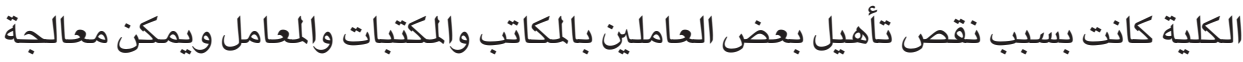

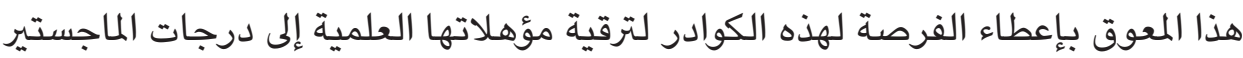

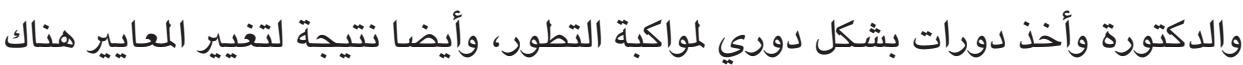

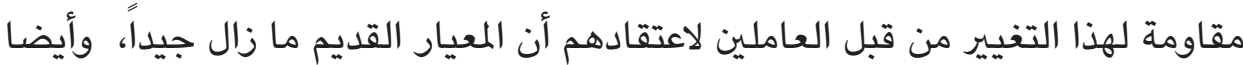
نقص ثقافة الجودة لدى كل من الطلاب والمدرسين والعاملين .

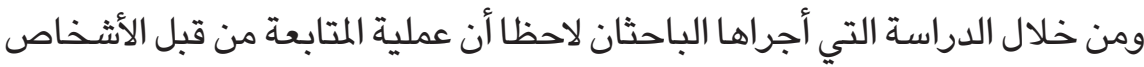

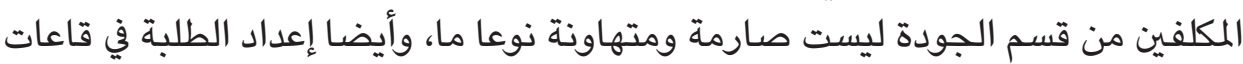
المحاضرات كبير بالنسبة لعدد المدرسين، وهذا قد يعيق الحصول على مخرجات ممتازة تتناسب مع المطالب الاقتصادية للبلاد.

\section{تطوير إدارة جودة التعليم في جامعت المحمديتمالانج:}

إن تحسين أداء مؤسسات التعليم الجامعي، يشكل اهتماما عالميا في جميع دول

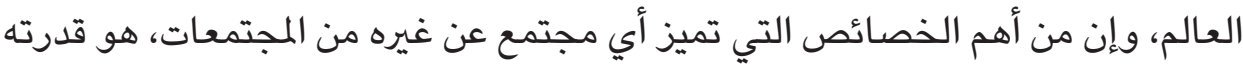

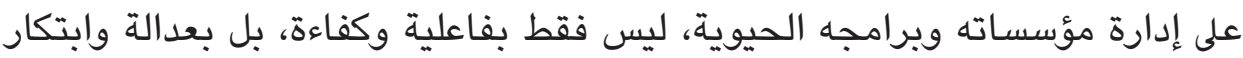

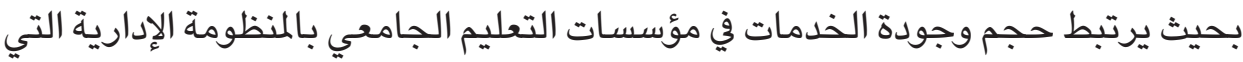


تجعل رسالة الجامعة بوصلة الحركة عن طريق المبادئ الإرشادية والأخلاق الجامعية،

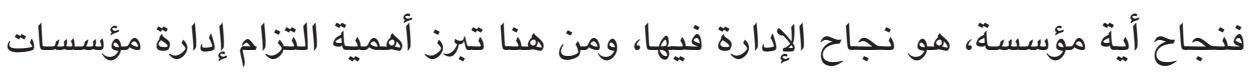

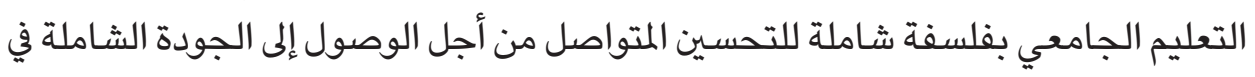

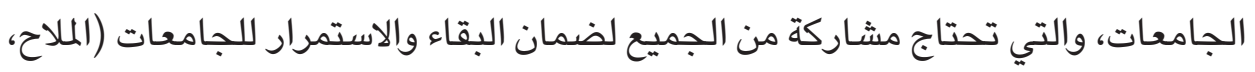

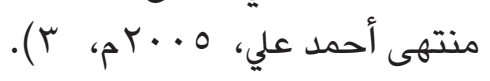
ومن خلال المقابلات تبين أنه يتم تطوير إدارة الجودة في كلية الدراسات الإسلامية

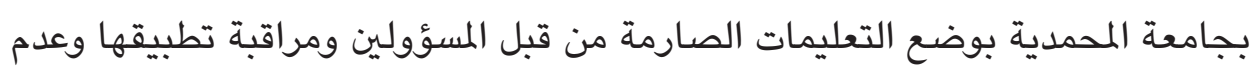

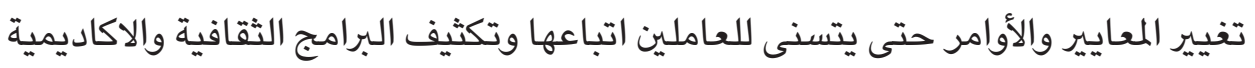

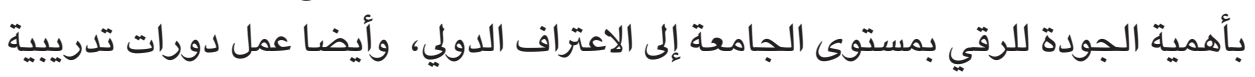
خاصة لرفع كفاءة العاملين بالكلية. ورأى الباحثان أن عملية التطوير تتم بصورة جيدة بدليل التطور الملحوظ للجامعة

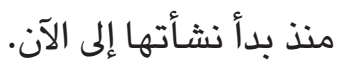

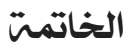

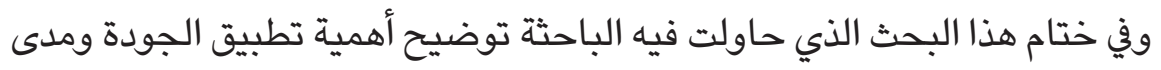

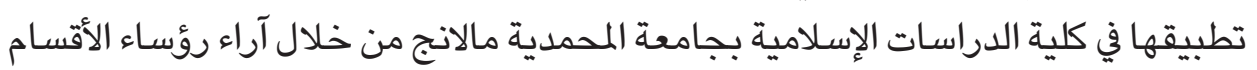

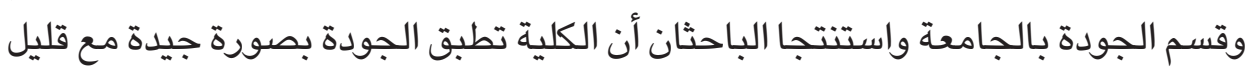
من القصور في بعض النواحي.

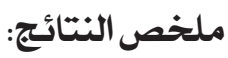

معايير الجودة في جامعة المحمدية مالانج كانت كبيرة وتتبع قوانين التربية العالية

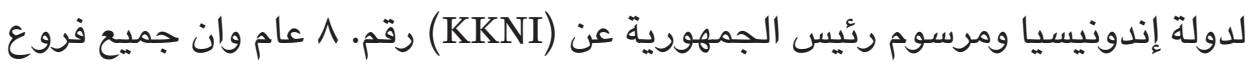

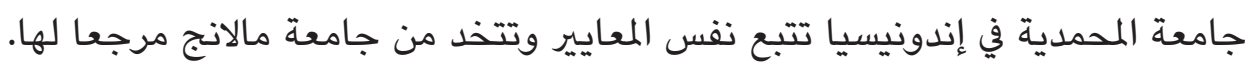

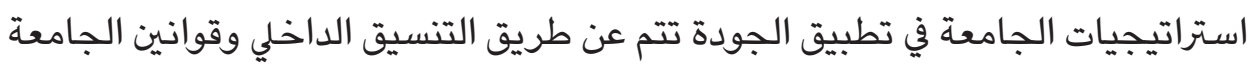
وتطبيق الاستراتيجية المركزية للإدارة.

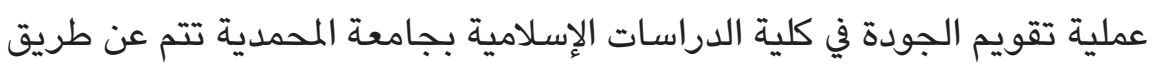
الملاحظة والتقييم المباشرة على أنشطة التعليم من خلال الدوائر التلفزيونية المغلقة والأدوات

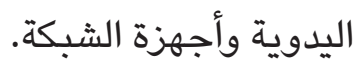


معوقات جودة التعليم في الكلية كانت بسبب نقص تأهيل بعض العاملين، وأيضا نتيجة لتغيير المعايير بصورة مستمرة.

يتم تطوير إدارة الجودة بوضع التعليمات الصارمة من قبل المسؤولين ومراقبة

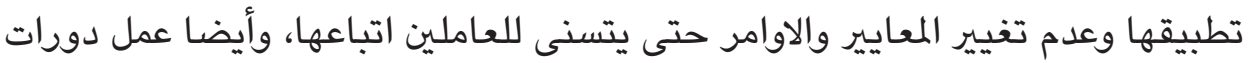
تدريبية خاصة لرفع كفاءة العاملين بالكلية .

المراجع والمصادر العربيتت

إبراهيم أنيس. المجمم الوسيط. د.ت. الطبعة الأولى. القاهرة: مجمع اللغة العربية . ابن منظور، جمال الدين محمد. ع/9 ام. لسان العرب. بيروت: دار صادر للطباعة والنشر. خالد أحمد الصرايرة، ليلى العساف، (إدارة الجودة الشاملة في مؤسسات التعليم العالي

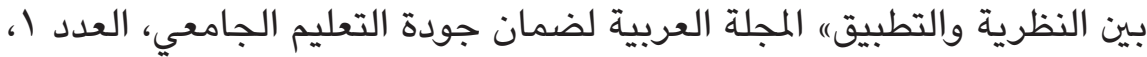

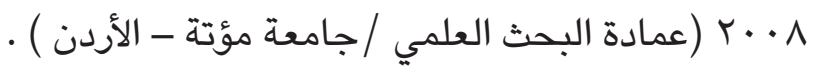

رجا، قدار. المدخل إلى إدارة الجودة الشاملة والإيزو . . .9 ـ 991 م، الطبعة الأولى. دمشق: دار الحصاد للنشر.

هيئة اعتماد مؤسسات التعليم العالي، دليل إجراءات معايير الجودة في مؤسسات التعليم

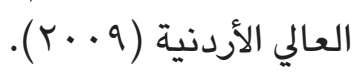

هند غسان أبو الشعر: معايير الجودة المعتمدة في مؤسسات التعليم العالي، الأردن، جامعة

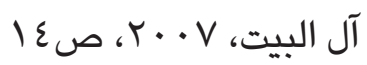

مصطفي أحمد (Y + - Y)، محمد الأنصاري، برنامج إدارة الجودة الشاملة وتطبيقاتها في المجال التربوي، قطر، المركز العربي للتدريب التربوي لدول الخليج..

الملاح، منتهى أحمد علي. 0 × · م. درجة تحقيق معايير إدارة الجودة الشاملة في الجامعات

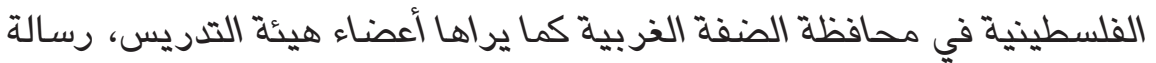
ماجستير، كلية الدراسات العليا، جامعة النجاح، فلسطين.

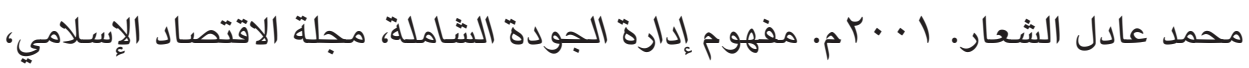

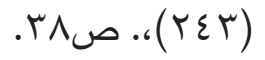


B.G. Dale. R.J. Boaden، M. Wilcox، R.E. McQuater، (1997) "Sustaining total quality management: what are the key issues?". The TQM Magazine. Vol. 9 Iss: 5. pp. $372-380$

http://kenanaonline.com/users/azhar-gaper/posts/136943

Peraturan Menteri Pendidikan dan Kebudayaan Nomor 73 Tahun 2013, Tentang Penerapan Kerangka Kualifikasi Nasional Indonesia Bidang Pendidikan Tinggi

Perpres RI No. 8 Tahun 2012 Tentang Kerangka Kualifikasi Nasional Indonesia (KKNI)

Permenristekdikti No. 14 Tahun 2015 Tentang SNPT (Standar Nasional Pendidikan Tinggi 